\title{
Supplemental Material for Bayesian Spatiotemporal Modeling using Hierarchical Spatial Priors, with Applications to Functional Magnetic Resonance Imaging
}

\author{
Martin Bezener John Hughes Galin Jones
}

\section{Posterior Sampling}

This section describes posterior sampling and estimation. The dimension of $q(\gamma, S, r, \rho \mid$ $y)$ is $p(N+G+1)+N$, so we are dealing with a high dimensional posterior in this problem. Sampling from high dimensional posteriors is challenging and componentwise sampling schemes (Johnson et al., 2013) are often employed to make the problem manageable. We adopt this approach here and so one complete update sequence of all the parameters looks like

$$
(S, \gamma, r, \rho) \rightarrow\left(S^{\prime}, \gamma, r, \rho\right) \rightarrow\left(S^{\prime}, \gamma^{\prime}, r, \rho\right) \rightarrow\left(S^{\prime}, \gamma^{\prime}, r^{\prime}, \rho\right) \rightarrow\left(S^{\prime}, \gamma^{\prime}, r^{\prime}, \rho^{\prime}\right) .
$$

We include this to be explicit about our algorithm. There has been a limited amount of study of the convergence properties of component-wise Metropolis-Hastings algorithms (Johnson et al., 2013; Jones et al., 2014), but we are unaware of any research on constructing an optimal update order.

\subsection{Sampling $S \mid \gamma, r, \rho, y$}

This sampling step involves $p G$ updates. Recall that $S=\left(S_{(1)}, S_{(2)}, \ldots, S_{(p)}\right)$, so we update $S$ according to the sequence

$$
\left(S_{(1)}, S_{(2)}, \ldots, S_{(p)}\right) \rightarrow\left(S_{(1)}^{\prime}, S_{(2)}, \ldots, S_{(p)}\right) \rightarrow \ldots \rightarrow\left(S_{(1)}^{\prime}, S_{(2)}^{\prime}, \ldots, S_{(p)}^{\prime}\right) .
$$

The $g$ components of each $S_{(j)}$ are updated as

$$
\underbrace{\left(S_{1, j}, S_{2, j}, \ldots, S_{G, j}\right)}_{S_{(j)}} \rightarrow\left(S_{1, j}^{\prime}, S_{2, j}, \ldots, S_{G, j}\right) \rightarrow \ldots \rightarrow \underbrace{\left(S_{1, j}^{\prime}, S_{2, j}^{\prime}, \ldots, S_{G, j}^{\prime}\right)}_{S_{(j)}^{\prime}} .
$$

To simplify notation, let $\gamma_{(g, j)}$ denote the vector of all $\gamma_{v, j}$ in region $g$ under task $j$.

$$
\pi\left(\gamma_{(g, j)} \mid S_{g, j}\right)=\prod_{v \in \mathcal{R}_{g}} \pi\left(\gamma_{v, j} \mid S_{g, j}\right) .
$$

Also let

$$
S_{(-g, j)}=\left(S_{1, j}^{\prime}, \ldots, S_{g-1, j}^{\prime}, S_{g+1, j}, \ldots, S_{G, j}\right)
$$


and

$$
S_{-g, j}=\left(S_{(1)}^{\prime}, \ldots, S_{(j-1)}^{\prime}, S_{(-g, j)}, S_{(j+1)}, \ldots, S_{(p)}\right) .
$$

The conditional density of interest is

$$
\begin{aligned}
q\left(S_{g, j} \mid S_{-g, j}, \gamma, r, \rho, y\right) & =q\left(S_{g, j} \mid S_{(-g, j)}, \gamma_{(j)}, r_{j}\right) \\
& \propto \pi\left(\gamma_{(g, j)} \mid S_{g, j}\right) \pi\left(S_{(j)} \mid r_{j}\right) .
\end{aligned}
$$

It is difficult to directly sample from (2). To obtain a draw, we employ a Metropoliswithin-Gibbs step. Let $p_{S}\left(S_{g, j}^{*} \mid S_{g, j}\right)$ denote the proposal density. The Hastings ratio is

$$
\alpha_{S_{(j)}^{*}}=\frac{\pi\left(\gamma_{(g, j)} \mid S_{g, j}^{*}\right) \pi\left(S_{(j)}^{*} \mid r_{j}\right) p_{S}\left(S_{g, j} \mid S_{g, j}^{*}\right)}{\pi\left(\gamma_{(g, j)} \mid S_{g, j}\right) \pi\left(S_{(j)} \mid r_{j}\right) p_{S}\left(S_{g, j}^{*} \mid S_{g, j}\right)}
$$

where

$$
S_{(j)}^{*}=\left(S_{1, j}^{\prime}, \ldots, S_{g-1, j}^{\prime}, S_{g, j}^{*}, S_{g+1, j}, \ldots, S_{G, j}\right)
$$

We use a random walk proposal $S_{g, j}^{*} \sim N\left(S_{g, j}, \sigma_{S_{g, j}}^{2}\right)$ where $S_{g, j}$ is the current state and $\sigma_{S_{g, j}}^{2}$ is a tuning paremeter chosen to achieve a desired acceptance rate. Under the random walk scheme, the Hastings ratio becomes

$$
\alpha_{S_{(j)}^{*}}=\frac{\pi\left(\gamma_{(v, j)} \mid S_{g, j}^{*}\right)\left[S_{(j)}^{* T} \Gamma_{j}^{-1} S_{(j)}^{*}\right]^{-G / 2}}{\pi\left(\gamma_{(v, j)} \mid S_{g, j}\right)\left[S_{(j)}^{T} \Gamma_{j}^{-1} S_{(j)}\right]^{-G / 2}} .
$$

\subsection{Sampling $\gamma \mid r, \rho, S, y$}

We now turn our attention to updating $\gamma$. This sampling step consists of $p N$ updates and is a two-step process. The $\gamma_{(j)}$ are updated in the following order:

$$
\left(\gamma_{(1)}, \gamma_{(2)}, \ldots, \gamma_{(p)}\right) \rightarrow\left(\gamma_{(1)}^{\prime}, \gamma_{(2)}, \ldots, \gamma_{(p)}\right) \rightarrow \ldots \rightarrow\left(\gamma_{(1)}^{\prime}, \gamma_{(2)}^{\prime}, \ldots, \gamma_{(p)}^{\prime}\right)
$$

We update the $N$ elements of each $\gamma_{(j)}$ one at a time. Therefore, one update of $\gamma_{(j)}$ looks like

$$
\underbrace{\left(\gamma_{1, j}, \gamma_{2, j}, \ldots, \gamma_{N, j}\right)}_{\gamma_{(j)}} \rightarrow\left(\gamma_{1, j}^{\prime}, \gamma_{2, j}, \ldots, \gamma_{N, j}\right) \rightarrow \ldots \rightarrow \underbrace{\left(\gamma_{1, j}^{\prime}, \gamma_{2, j}^{\prime}, \ldots, \gamma_{N, j}^{\prime}\right)}_{\gamma_{(j)}^{\prime}} .
$$

The conditional density of interest is

$$
q\left(\gamma_{v, j} \mid \gamma_{-v, j}, S_{g, j}, y\right) \propto \pi\left(\gamma_{v, j} \mid S_{g, j}\right)\left(1+T_{v}\right)^{-\gamma_{v, j} / 2} K\left(\rho_{v}, \gamma_{v}\right)^{-T_{v} / 2}
$$

where

$$
\gamma_{-v, j}=\left(\gamma_{1, j}^{\prime}, \ldots, \gamma_{v-1, j}^{\prime}, \gamma_{v+1, j}, \ldots, \gamma_{N, j}\right) .
$$

We directly sample $\gamma_{v, j}$ by simply evaluating (3) at $\gamma_{v, j}=0$ and $\gamma_{v, j}=1$ and normalizing. 


\subsection{Sampling $r \mid \rho, S, \gamma, y$}

Recall that $r=\left(r_{1}, r_{2}, \ldots, r_{p}\right)$. One update of $r$ looks like

$$
\left(r_{1}, r_{2}, \ldots, r_{p}\right) \rightarrow\left(r_{1}^{\prime}, r_{2}, \ldots, r_{p}\right) \rightarrow \ldots \rightarrow\left(r_{1}^{\prime}, r_{2}^{\prime}, \ldots, r_{p}^{\prime}\right) .
$$

First, notice that

$$
q\left(r_{j} \mid r_{-j}, \rho, S, \gamma, y\right)=\pi\left(S_{(j)} \mid r_{j}\right) \pi\left(r_{j}\right) .
$$

It is difficult to directly sample from (5), so we employ a Metropolis-within-Gibbs step to obtain a draw. Let $p_{r}\left(r_{j}^{*} \mid r_{j}\right)$ denote the proposal density. The Hastings ratio is

$$
\alpha_{r_{j}^{*}}=\frac{\pi\left(S_{(j)} \mid r_{j}^{*}\right) \pi\left(r_{j}^{*}\right) p_{r}\left(r_{j} \mid r_{j}^{*}\right)}{\pi\left(S_{(j)} \mid r_{j}\right) \pi\left(r_{j}\right) p_{r}\left(r_{j}^{*} \mid r_{j}\right)} .
$$

Once again we use a random walk proposal $r_{j}^{*} \sim N\left(r_{j}, \sigma_{r_{j}}^{2}\right)$, where $r_{j}$ is current value and $\sigma_{r_{j}}^{2}$ is chosen to achieve the desired acceptance rate. In this case, the Hastings ratio becomes

$$
\alpha_{r_{j}^{*}}=\frac{\left|\Gamma_{j}^{*}\right|^{-1 / 2}\left[S_{(j)}^{T} \Gamma_{j}^{*-1} S_{(j)}\right]^{-G / 2} \pi\left(r_{j}^{*}\right)}{\left|\Gamma_{j}\right|^{-1 / 2}\left[S_{(j)}^{T} \Gamma_{j}^{-1} S_{(j)}\right]^{-G / 2} \pi\left(r_{j}\right)},
$$

where $\Gamma_{j}^{*}$ is the correlation matrix in (6) evaluated at $r_{j}^{*}$. This step is computationally expensive due to the inversion of $\Gamma_{j}^{*}$. Additionally, to avoid issues with numerical underflow, it is safer to work with the $\log$ determinant of $\Gamma_{j}^{*}$, which is also an expensive operation.

\subsection{Sampling $\rho \mid S, \gamma, r, y$}

Recall that $\rho=\left(\rho_{1}, \rho_{2}, \ldots, \rho_{N}\right)$. In this step, the $N$ correlation coefficients are updated using the following scheme:

$$
\left(\rho_{1}, \rho_{2}, \ldots, \rho_{N}\right) \rightarrow\left(\rho_{1}^{\prime}, \rho_{2}, \ldots, \rho_{N}\right) \rightarrow \ldots \rightarrow\left(\rho_{1}^{\prime}, \rho_{2}^{\prime}, \ldots, \rho_{N}^{\prime}\right) .
$$

The conditional posterior of each $\rho_{v}$ is

$$
\begin{aligned}
q\left(\rho_{v} \mid \rho_{-v}, S, \gamma, r, y\right) & \propto q\left(\rho_{v} \mid \gamma_{v}, y\right) \\
& \propto \pi\left(\rho_{v}\right)\left|\Lambda_{v}\right|^{-1 / 2} K\left(\rho_{v}, \gamma_{v}\right)^{-T_{v} / 2} .
\end{aligned}
$$

Since direct sampling from (6) is not feasible, we use a Metropolis-within-Gibbs step here. Let $p_{\rho}\left(\rho_{v}^{*} \mid \rho_{v}\right)$ be the proposal distribution. The Hastings ratio is

$$
\alpha_{\rho_{v}^{*}}=\frac{\pi\left(\rho_{v}^{*}\right)\left|\Lambda_{v}^{*}\right|^{-1 / 2} K\left(\rho^{*}, \gamma_{v}\right)^{-T_{v} / 2} p_{\rho}\left(\rho_{v} \mid \rho_{v}^{*}\right)}{\pi\left(\rho_{v}\right)\left|\Lambda_{v}\right|^{-1 / 2} K\left(\rho, \gamma_{v}\right)^{-T_{v} / 2} p_{\rho}\left(\rho_{v}^{*} \mid \rho_{v}\right)},
$$


where $\Lambda_{v}^{*}(i, k)=\rho_{v}^{*|i-k|}$ and

$$
\rho^{*}=\left(\rho_{1}^{\prime}, \ldots, \rho_{v-1}^{\prime}, \rho_{v}^{*}, \rho_{v+1}, \ldots, \rho_{N}\right) .
$$

We use an independence sampler with a $U(-1,1)$ proposal distribution, in which case the Hastings ratio becomes

$$
\alpha_{\rho_{v}^{*}}=\frac{\pi\left(\rho_{v}^{*}\right)\left|\Lambda_{v}^{*}\right|^{-1 / 2} K\left(\rho^{*}, \gamma_{v}\right)^{-T_{v} / 2}}{\pi\left(\rho_{v}\right)\left|\Lambda_{v}\right|^{-1 / 2} K\left(\rho, \gamma_{v}\right)^{-T_{v} / 2}} .
$$

This step is computationally intensive since one update of $\rho$ requires inverting $N$ matrices with dimension $T_{v}$. This step is avoided if the empirical Bayes prior is used.

\subsection{Starting Values}

Selection of initial values for the simulation is important when faced with high-dimensional posterior distributions. Bezener et al. (2016) suggest that an efficient way to choose starting values is to perform a preliminary frequentist analysis (with, say, the SPM software) and set the initial values of the simulation as follows.

(1.) Set each $\gamma_{v, j}^{(0)}=\hat{\gamma}_{v, j}^{\text {freq }}$.

(2.) Initialize each spatial random effect as $S_{g, j}^{(0)}$ by first computing

$$
\hat{\pi}_{g, j}=\frac{1}{n_{g}} \sum_{v \in \mathcal{R}_{g}} \hat{\gamma}_{v, j}^{\text {freq }}
$$

where $n_{g}$ is the number of voxels in the $g$ th parcel and then solving (4) in the main paper to get

$$
S_{g, j}^{(0)}=\log \left(\frac{\hat{\pi}_{g, j}}{1-\hat{\pi}_{g, j}}\right)
$$

(3.) Use a variogram with the $S_{g, j}^{(0)}$ from the previous step to determine $r_{j}^{(0)}$.

An advantage of choosing a good initial value is that we can avoid the use of burn-in. This leads to more efficient use of the MCMC samples and reduces the variance of the posterior mean estimates (Bezener et al., 2016; Flegal et al., 2008; Flegal and Jones, 2011; Geyer, 2011; Jones and Hobert, 2001)

\section{Additional Simulation Results}

We now investigate the validity of the proposed activation threshold through a simulation experiment. We reclassify activation in the 15 simulated data sets from Sections 3.1 and 3.2 of the main paper which were generated when $r=8$ at both $\beta_{v, 1}=3$ and 5 . The 
following three activation thresholds are considered: $0.7946,0.8722$, and 0.9650 . These values correspond to $p$-values of 0.1, 0.05, and 0.01, respectively (Lee et al., 2014; Smith and Fahrmeir, 2007) with correpsonding upper bounds on the false discovery rate of $0.2054,0.1278$, and 0.035 , respectively. The results are reported in Table 1 . Overall, we conclude that our recommended activation threshold works well as it provides a nice balance between accuracy and FPR.

\begin{tabular}{ccc}
\hline Activation Threshold & Accuracy (\%) & FPR (\%) \\
\hline 0.7946 & $91.47(0.07)$ & $2.30(0.04)$ \\
0.8722 & $90.00(0.08)$ & $1.20(0.03)$ \\
0.9650 & $84.92(0.09)$ & $0.61(0.01)$ \\
\hline
\end{tabular}

(a)

\begin{tabular}{ccc}
\hline Activation Threshold & Accuracy (\%) & FPR (\%) \\
\hline 0.7946 & $97.64(0.04)$ & $3.59(0.03)$ \\
0.8722 & $97.88(0.04)$ & $2.04(0.03)$ \\
0.9650 & $96.65(0.05)$ & $1.00(0.02)$ \\
\hline
\end{tabular}

(b)

Table 1: Classification accuracies and false positive rates at various activation thresholds when (a) $\beta_{v, 1}=3$ and (b) $\beta_{v, 1}=5$ and the $\chi_{8}^{2}$ prior is used for $r$.

We conclude this section by checking the performance of our method under a longer simulated experiment. The simulation in Section 3.1 of the main paper considers a single block experiment, which is adequate for the purposes of exploring methodology performance, but not representative of an actual fMRI experiment. We consider the design matrix in Figure 1, which includes 3 replications of a task block. We use the same settings as the simulation in Section 3.1 of the paper to generate the BOLD signal, although we only consider $r=8$ and a $\chi_{8}^{2}$ prior. We consider $\beta_{v, 1}=0.1,0.5,1,3$, and 5 .

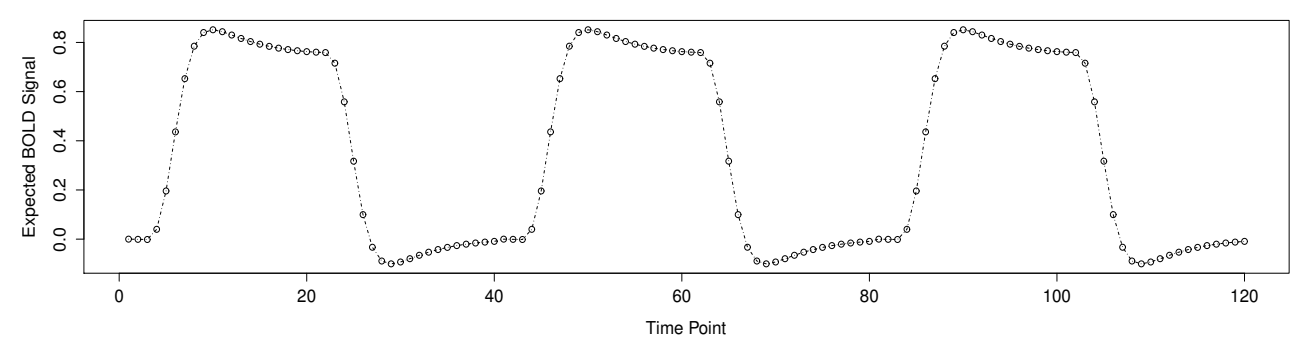

Figure 1: A design matrix representative of a blocked experiment with three replications.

The classification accuracy and false positive rate (FPR) results are in Table 2. As 


\begin{tabular}{cccc}
\hline$\beta_{1, v}$ & Accuracy (\%) & FPR (\%) & SB Accuracy (\%) \\
\hline 0.1 & $49.25(0.09)$ & $0.69(0.05)$ & - \\
0.5 & $57.88(0.09)$ & $0.59(0.02)$ & - \\
1 & $71.54(0.07)$ & $0.59(0.02)$ & - \\
3 & $97.67(0.05)$ & $0.60(0.01)$ & $90.00(0.08)$ \\
5 & $99.58(0.03)$ & $0.66(0.01)$ & $97.88(0.04)$ \\
\hline
\end{tabular}

Table 2: Classification accuracies and false positive rates at various $\beta_{v, 1}$ under a longer simulated experiment.

a reference, we also put the corresponding simulation results from the single block (SB) experiment under identical settings. It is obvious that the addition of the two extra blocks substantially improves the performance, both in terms of classification accuracy, and false positive rate.

\section{Emotion Data Example Diagnostics}

We checked trace plots of the spatial dependence parameters and a subset of 30 randomly selected spatial random effects under both tasks. Figure 2 displays the plots of three spatial random effects and the spatial dependence parameter $r$ under both the face and shape blocks. There are no obvious signs of convergence problems.

We also consider voxel-level diagnostics to assess model fit. We checked the assumption of Gaussian errors, as well as the adequacy of the AR(1) error structure, at 30 randomly selected voxels. Four randomly selected voxels are displayed in Figure 3 . The first row displays the fitted regression line (in red) as well as the observed BOLD signal. The leftmost plot is a voxel that was active only under the face task. The middle two plots are voxels that were active under both tasks, and the rightmost plot is a voxel that was not active under either task. The second row shows the quantile-quantile plots, which do not indicate any problems. Finally, the autocorrelation plots of the adjusted residuals are shown in the third row. The plots do not suggest that a higher-order autoregressive process is necessary to model the residual autocorrelation.

\section{Second Data Example}

We analyze the auditory data from the Wellcome Trust Centre for Neuroimaging using our proposed method. The original data set can be found at www.fil.ion.ucl.ac.uk/ spm/data/auditory/ .

\subsection{Description of the Experiment}

A single subject was studied in this experiment. Before data collection, the image space was partitioned into a $64 \times 64 \times 64$ rectangular lattice with voxel size $3 \mathrm{~mm}^{3}$. During the experiment, a total of 84 scans were collected on a $2 \mathrm{~T}$ Siemens scanner, with one scan 

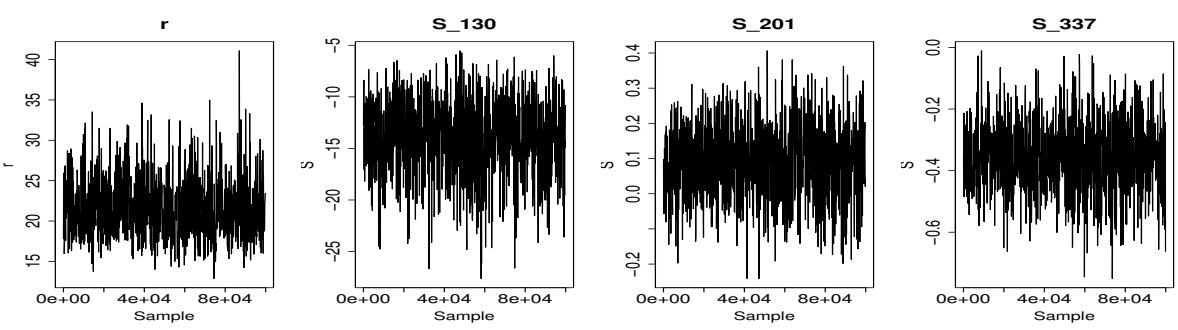

(a)
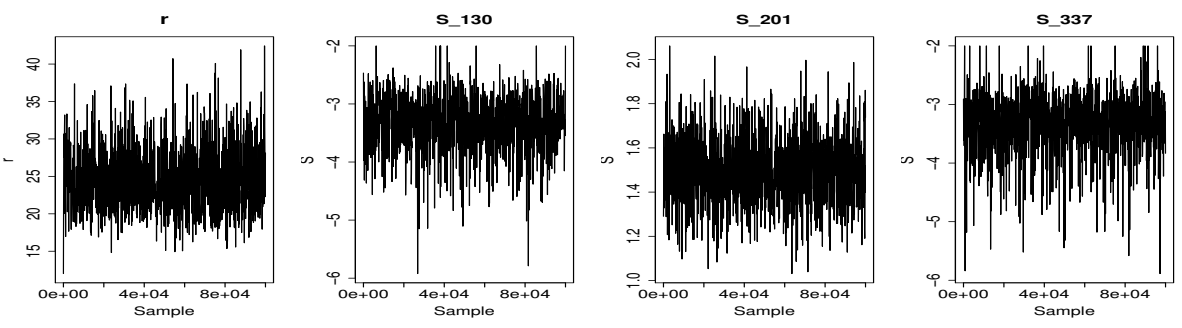

(b)

Figure 2: Trace plots of the spatial dependence parameter $r$ and three randomly selected spatial random effects $S_{g, j}$ under the (a) shape blocks and (b) face blocks in the HCP analysis.

taken every seven seconds. The experiment was constructed as a block design using 14 blocks of six scans, with each block lasting 42 seconds.

During the odd-number blocks, the subject rested. During the even number blocks, the subject listened to bi-syllabic words presented in rapid succession. The goal was to determine regional activations during the experiment. Before statistical analysis, we preprocessed the data using standard techniques and partitioned the voxels into 400 contiguous anatomical regions. Because we expected activation to mostly occur in the parietal lobe and possibly the frontal lobe, we used k-means clustering to cluster the voxels in the top $143 \mathrm{~mm}$-thick vertical slices into 330 regions. The remaining space was clustered into 70 regions.

Since we expected a significant amount of spatial dependence in the images, we chose a $\chi_{12}^{2}$ prior for $r$. The design matrix used in this experiment is displayed in Figure 4 and was obtained by convolving the canonical hemodynamic response function with the stimulus function as described in Section 2.1 of the main paper.

\subsection{Computation}

We drew 150,000 MCMC samples from the posterior distribution in (9) of the main paper. All tuning parameters were selected to achieve acceptance rates of approximately 


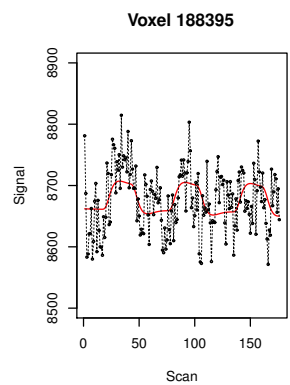

Voxel 188395

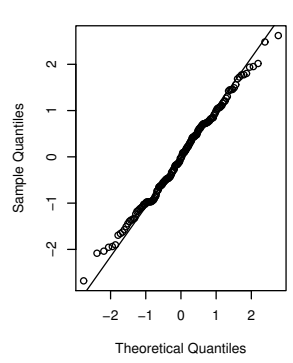

Voxel 188395

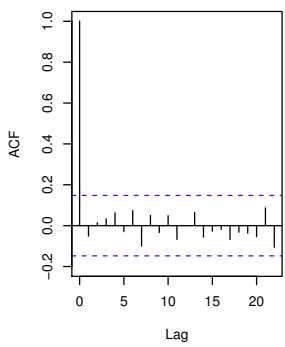

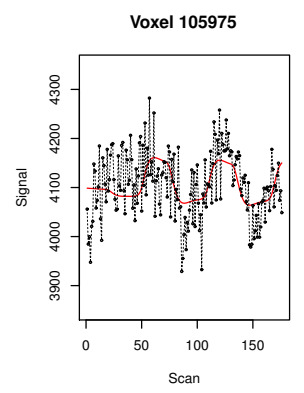

Voxel 105975

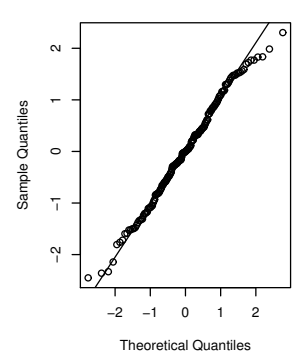

Voxel 105975

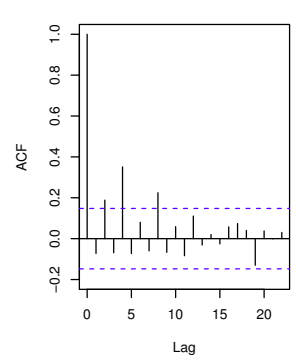

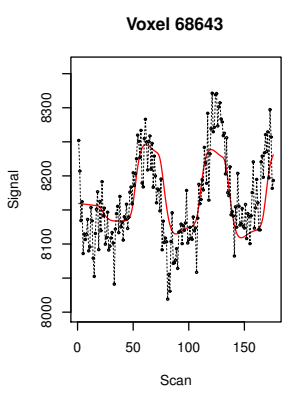

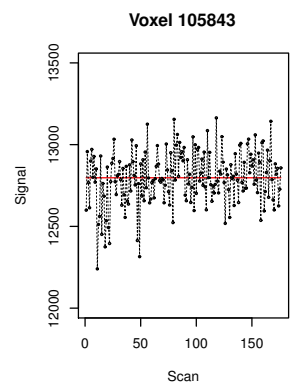

Voxel 68643

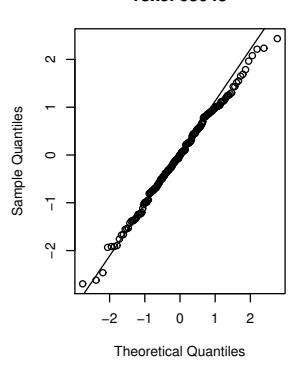

Voxel 68643

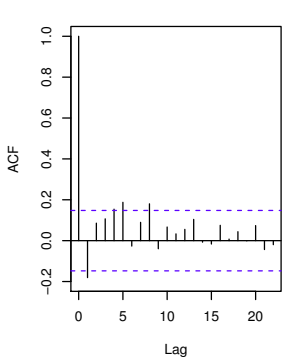

Voxel 105843

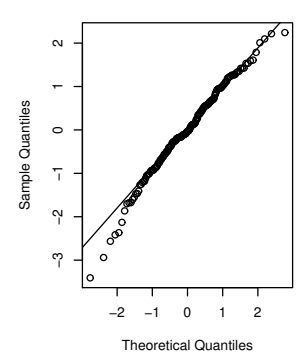

Voxel 105843

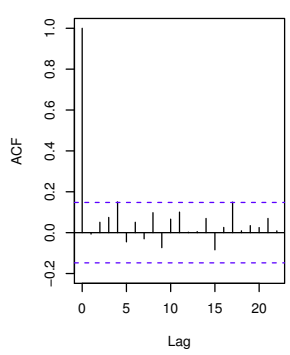

Figure 3: Diagnostic plots of 4 randomly selected voxels in the HCP data analysis.

$50 \%$ in the Metropolis-Hastings steps. Standard errors were all less than $2 \%$ of their posterior estimates and are therefore omitted in this section. We also checked the trace plots of the spatial random effects $S$ and spatial correlation parameters $r$, since those were sampled using a Metropolis-within-Gibbs step. We checked 20 trace plots for convergence issues, and Figure 5 displays the plots of three randomly selected spatial random effects. Figure 6 displays the trace plot of $r$. There are no obvious signs of convergence problems.

We note that this method took less than 5 hours on a single core of an Intel i7-4770 $3.5 \mathrm{GHz}$ processor, which shows that it is computationally reasonable in practice. 


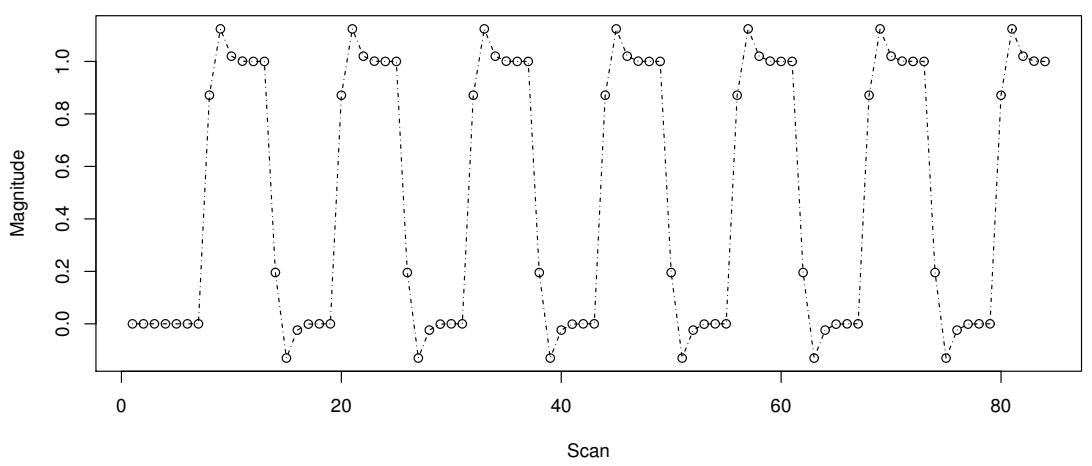

Figure 4: The design matrix used in the auditory data analysis.

\subsection{Results}

The results of four consecutive vertical slices showing the bulk of the activation are displayed in Figure 7. Using an activation threshold of 0.8722 , our method classified $2.6 \%$ of voxels as activated during the listening blocks. Most of the activation occurs in the parietal lobe, which is expected since it is responsible for processing auditory information. A small cluster of activity is also detected in the frontal lobe.

The posterior estimate of the spatial correlation parameter is $\hat{r}=20.18$, indicating a very high amount of spatial correlation in the images. This posterior estimate agrees with the voxel activation patterns displayed in Figure 7 as well as what is expected scientifically.
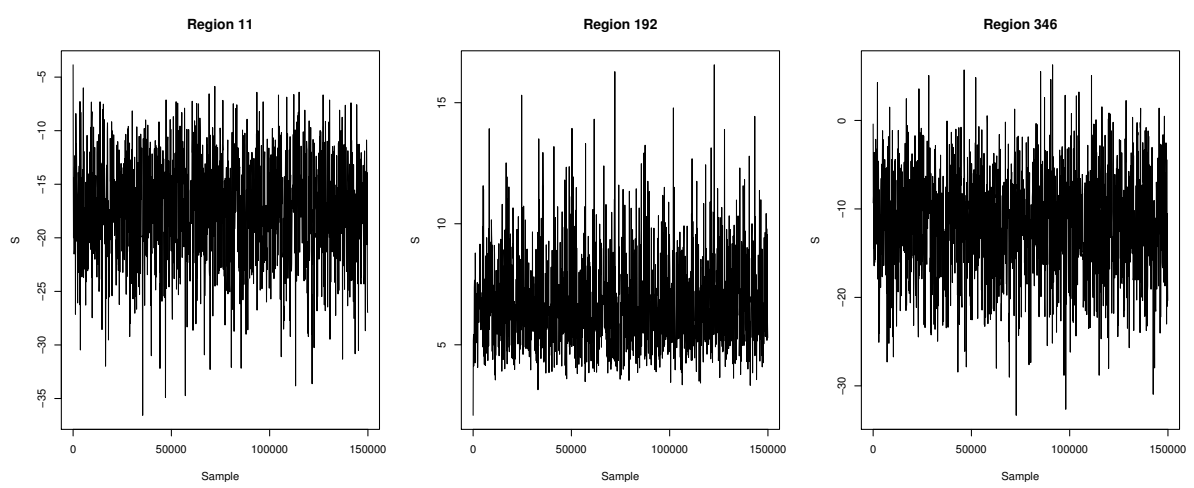

Figure 5: The trace plots of three spatial random effects $S$. Every 100th point is plotted. 


\begin{tabular}{cc}
\hline Threshold & Active Voxels (\%) \\
\hline 0.7946 & 2.94 \\
0.8722 & 2.66 \\
0.9650 & 2.14 \\
\hline
\end{tabular}

Table 3: The amount of activation detected at various activation thresholds.

The maximum likelihood estimates of the $\rho_{v}$ in four different slices are displayed in Figure 8. We notice that different regions exhibit different temporal correlation strengths.

\subsection{Model Validation}

We now assess the model fit. First, we investigated the amount of activation detected at the two alternative activation thresholds considered in Section 2 and report the results in Table 3. The amount of activation detected is similar under the three thresholds, and so our choice of 0.8722 appears to be reasonable.

Next, we turn our attention to regression diagnostics. We checked the assumption of normal errors, as well as the adequacy of the AR(1) error structure at 20 voxels. Two randomly selected active and inactive voxels are displayed in Figure 9. The first row displays the fitted regression line as well as the observed BOLD signal. It is obvious that the signal is noisy, which is common in fMRI. The second row contains the residual normality plots. Finally, the autocorrelation plots of the adjusted residuals are in third row. The plots do not suggest that a higher order autoregressive process is necessary to model the residual autocorrelation.

As a final note, we checked the stability of our procedure under different parcellations. We refit the model choosing all 400 region centroids completely at random over

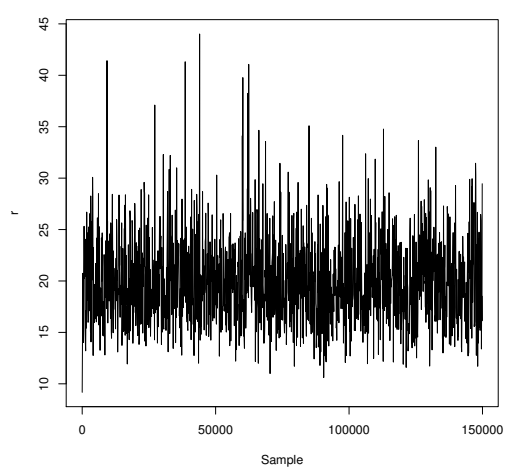

Figure 6: The trace plots of the spatial correlation parameter $r$. Every 100th point is plotted. 

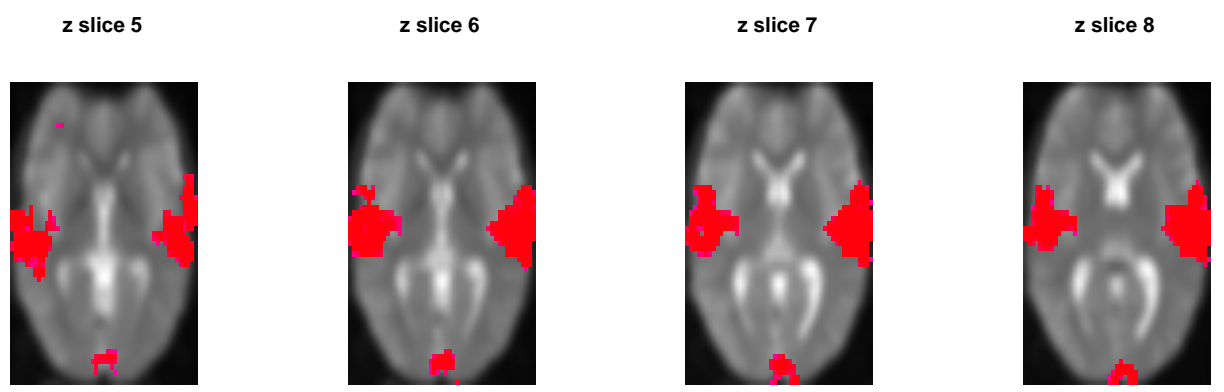

Figure 7: Active voxels in four consecutive vertical slices.
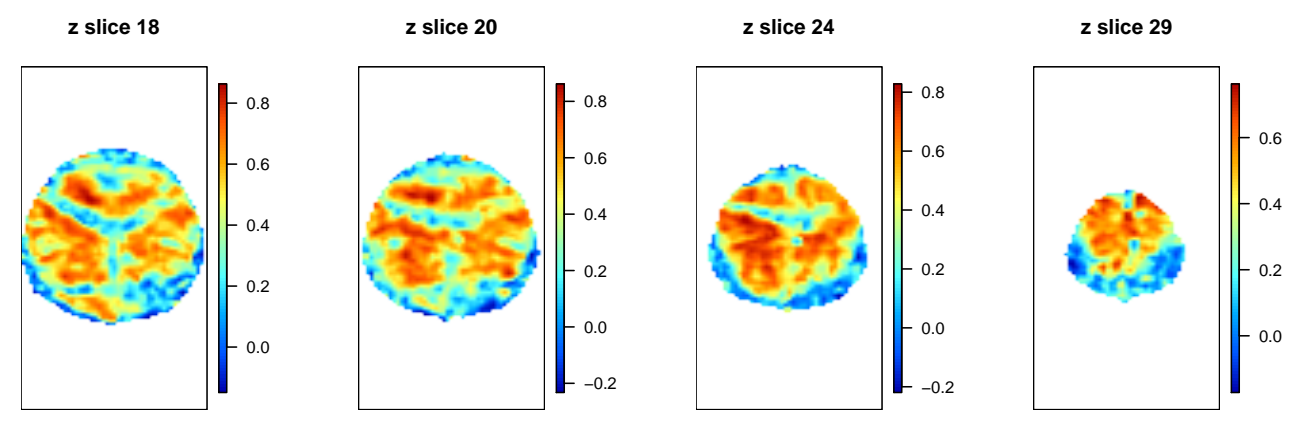

Figure 8: Maximum likelihood estimates of the $\operatorname{AR}(1)$ coefficients at four vertical slices.

the entire image. We then repeated this using 500 and 600 regions. All results were similar, although using finer parcellations will increase the computing time.

\section{References}

Bezener, M., Eberly, L. E., Hughes, J., Jones, G., and Musgrove, D. R. (2016). "Bayesian Spatiotemporal Modeling for Detecting Neuronal Activation via Functional Magnetic Resonance Imaging." In Härdle, W. K., Lu, H. H.-S., and Shen, X. (eds.), Handbook of Big Data Analytics, Springer Handbooks of Computational Statistics. Springer. 4

Flegal, J. M., Haran, M., and Jones, G. L. (2008). "Markov Chain Monte Carlo: Can We Trust the Third Significant Figure?" Statistical Science, 23: 250-260. 4

Flegal, J. M. and Jones, G. L. (2011). "Implementing Markov chain Monte Carlo: Estimating with confidence." In Brooks, S., Gelman, A., Jones, G., and Meng, X. (eds.), Handbook of Markov Chain Monte Carlo, 175-197. Chapman \& Hall/CRC Press. 4 

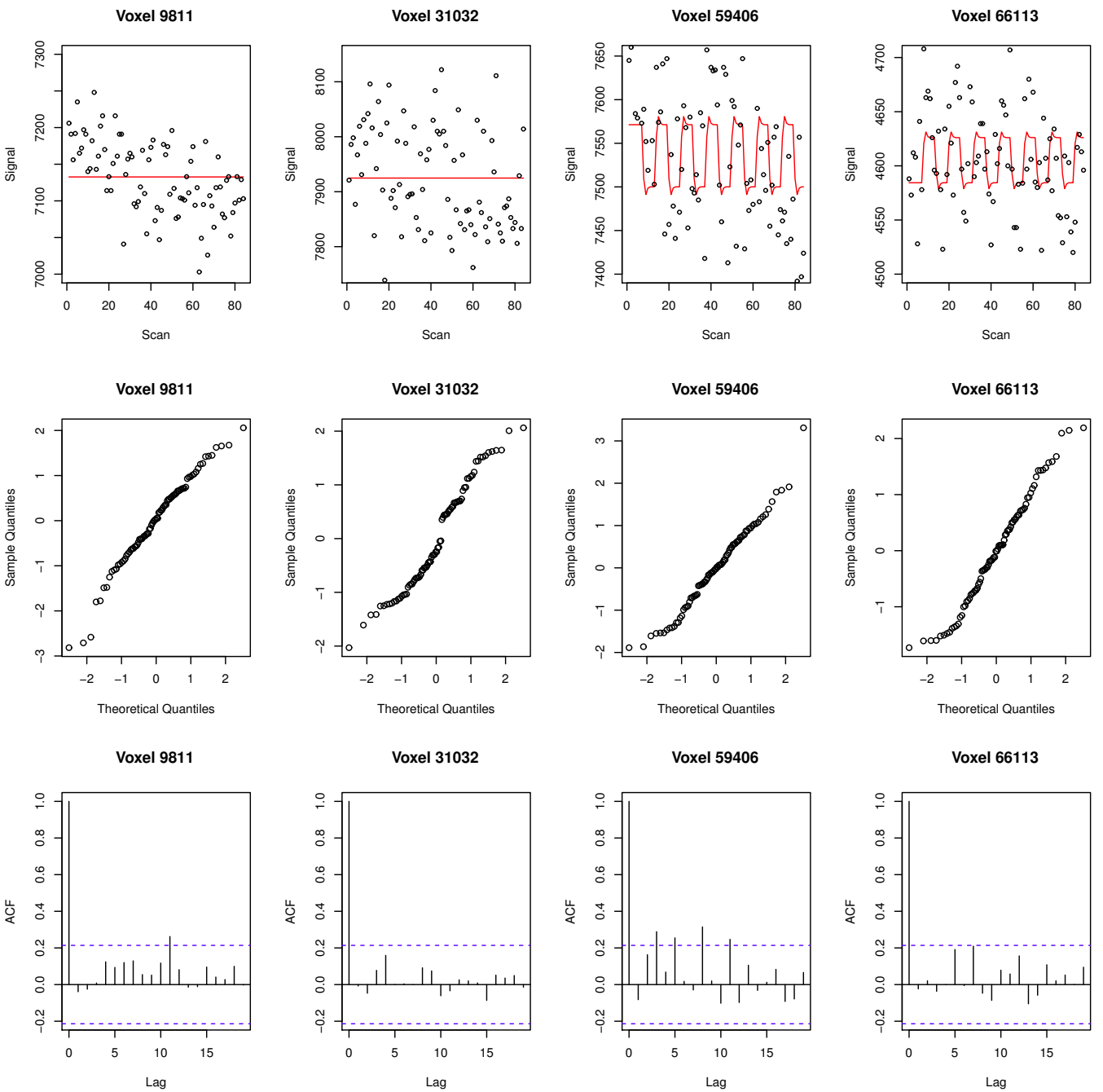

Figure 9: Diagnostic plots of 4 randomly selected voxels

Geyer, C. J. (2011). "Introduction to MCMC." In Brooks, S. P., Gelman, A. E., Jones, G. L., and Meng, X. L. (eds.), Handbook of Markov Chain Monte Carlo, 1-77. Chapman and Hall/CRC. 4

Johnson, A. A., Jones, G. L., and Neath, R. C. (2013). "Component-wise Markov chain Monte Carlo: Uniform and geometric ergodicity under mixing and composition." Statistical Science, 28: 360-375. 1

Jones, G. L. and Hobert, J. P. (2001). "Honest exploration of intractable probability distributions via Markov chain Monte Carlo." Statistical Science, 16: 312-334. 4 
Jones, G. L., Roberts, G. O., and Rosenthal, J. S. (2014). "Convergence of conditional Metropolis-Hastings samplers." Advances in Applied Probability, 46: 422-445. 1

Lee, K.-J., Jones, G. L., Caffo, B. S., and Bassett, S. S. (2014). "Spatial Bayesian Variable Selection Models on Functional Magnetic Resonance Imaging Time-Series Data." Bayesian Analysis, 9: 699-732. 5

Smith, M. and Fahrmeir, L. (2007). "Spatial Bayesian Variable Selection with Application to Functional Magnetic Resonance Imaging." Journal of the American Statistical Association, 102: 417-431. 5 